\title{
Systematisches Screening auf Distress: nicht mehr Supportivtherapie
}

\begin{abstract}
Ein Screening auf Distress bei onkologischen Patienten soll deren bislang nicht erkannte Bedürfnisse identifizieren. Aber: Werden die Patienten überwiesen? Kommt das Team für Supportivtherapie (SCT) dadurch ins Spiel?
\end{abstract}

n einem Monat wurden 934 Screening bögen onkologischer Patienten an einem großen US-Tumorzentrum gesammelt und die Krankendaten nach folgenden Kriterien analysiert: Wie oft finden sich Hinweise auf krankheitsbedingten Distress? Welcher Anteil der Patienten mit Distress wird an Mitglieder des SCT überwiesen? Wie häufig wird schließlich eine supportive Therapie durchgeführt?

Von 644 Patienten mit vollständig ausgefüllten Bögen berichteten 195 (30\%) über deutliche Distress-Symptome. 34 lehnten von vorneherein einen weiteren Kontakt ab, für 161 kam eine Weiterleitung an ein Mitglied des SCT infrage, je nach Art des Problems in den Psychologie-Bereich $(\mathrm{n}=77)$, zu einem Ernährungsberater $(n=97)$ oder einem Sozialarbeiter $(\mathrm{n}=71)$. Binnen 14 Tagen nach dem Screening konnte das initiale Assessment bei 99 Patienten komplettiert werden, bei den übrigen kam die Komplettierung aus unterschiedlichen Gründen nicht zustande. Zuletzt nahmen nur 19 der 99 Patienten zumindest einen Nachfolgetermin der Supportivtherapie wahr. Dabei war es egal, ob die Patienten früher bereits eine maligne Erkrankung hatten oder derzeit eine Tumortherapie stattfand oder nicht.

Das Screening scheint nicht zu einer verstärkten Nutzung von supportiven Therapieangeboten zu führen. Die Ursachen dieses Paradoxons sind noch nicht geklärt. Möglicherweise gibt es Zugangsbarrieren. Eine Fehlinterpretation könnte auch dadurch bedingt sein, dass in dieser Analyse nur Screening-basierte Überweisungen registriert wurden. Die Patienten konnten an dem Tumorzentrum aber auch direkt durch Überweisung der betreuenden Ärzte oder eigene Anfrage Dienste des SCT nutzen.

Fazit: Die Ergebnisse dieser Studie belegen einen erheblichen Bedarf onkologischer Patienten an Supportivtherapie. Andererseits führt das Screening offen- bar nicht zu einer erhöhten Nutzung solcher Angebote. Brigitte Schalhorn

Funk $R$ et al. What happens after distress screening? Patterns of supportive care service utilization among oncology patients identified through a systematic screening protocol. Support Care Cancer. 2016;24(7):2861-8.

\section{Kommentar von Prof. Herschbach:}

Krebspatienten können heute eine bedarfsgerechte psychoonkologische Unterstützung erwarten, wie im Nationalen Krebsplan konsentiert [Herschbach P, Mandel T. Onkologe. 2011;17(12):1107-14], in den DKG-Kriterien für die Zertifizierung onkologischer Zentren festgeschrieben [www.onkozert.de/ onkologische_zentren.htm] und in den S3Leitlinien Psychoonkologie hergeleitet [AWMF-Registernummer: 032/051OL]. Der Hintergrund hierfür ist, dass psychoonkologische Behandlungen ihre Wirksamkeit demonstrieren konnten. Sie verlängerten nicht die Überlebenszeit, unterstützten aber die Krankheitsbewältigung und die Lebensqualität. Zudem helfen sie, psychischen Störungen vorzubeugen, die in einer Studie bei $31,8 \%$ der Patienten dokumentiert wurden [Mehnert A et al. J Clin Oncol. 2014; 32(31):3540-6].

Das Problem in der Versorgung ist der Terminus bedarfsgerecht. Bei wem besteht Bedarf für psychoonkologische Versorgung? Wer stellt inn wie fest? In den letzten Jahrzehnten zeigte sich, dass weder die Selbstselektion der Patienten noch die Spontaneinschätzung der behandelnden Ärzte funktionieren. Die Folge: Eine unbefriedigende Unterversorgung [Carlson LE et al. Br J Cancer. 2004;90(12):2297-304].

Vor diesem Hintergrund wurde national und international die Empfehlung konsentiert, bei neu aufgenommenen Patienten mithilfe von Kurzfragebögen "distress screenings" durchzuführen. Überschreiten die Ergebnisse einen definierten Belastungssschwellenwert soll die Zuweisung psychosozialer Maßnahmen erfolgen.

Rachel Funk und Kollegen kommen zu dem Schluss, dass nur ein kleiner Teil der ge- screenten und belasteten Patienten die angebotene Unterstützung in Anspruch nimmt, und fragen nach den Gründen.

Die Studie ist in mancher Hinsicht nicht typisch für deutsche Verhältnisse. Nur ambulante Patienten wurden eingeschlossen, ein wenig verbreitetes Screeninginstrument kam zum Einsatz, das "distress screening" bezog sich differenziell auf die psychischen Bereiche Angst und Depression sowie Ernährung und Sozialberatung. Untersucht wurde ein zweistufiges Verfahren (ErstScreening und Folgekontakte). Es wird von einem Supportive Care Team gesprochen (das für die Patienten womöglich nicht als Team wahrgenommen wurde) und eher unpersönlich kommuniziert. Schade, dass die Forscher nicht die Art der Kommunikation und die Zufriedenheit mit dem Erstkontakt als Prädiktor analysiert haben.

\section{"Für somatisch Kranke ist es höchst kompliziert, psychoonkologische Therapie in Anspruch zu nehmen."}

Trotz dieser Einschränkungen adressieren Funk und Kollegen ein Phänomen, das auch uns Kopfzerbrechen bereitet. Wir wissen aus zahlreichen Studien, dass lediglich 20-40\% der psychisch hoch belasteten Krebspatienten psychosoziale Angebote in Anspruch nehmen [Dilworth $S$ et al. Psychooncology. 2014;23(6):601-12]. Die Ursachen sind vielfältig und können nach Gründen (Informationsmangel, Scham), soziodemografischen und medizinischen Bedingungen (männliches Geschlecht, Zeitpunkt im Krankheitsverlauf) und psychologischen Konstrukten (Neurotizismus, Depression) unterschieden werden. Hier ist noch viel Forschung erforderlich. Klar sollte sein, dass es für somatisch kranke Menschen ein höchst kompliziertes Unterfangen ist, psychoonkologische Therapie in Anspruch zu nehmen.

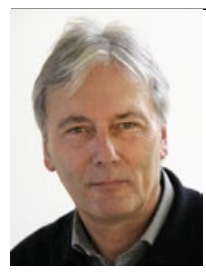

Prof. Dr. rer soc. Peter Herschbach Direktor des RomanHerzog Krebszentrums am Klinikum rechts der Isar der TU München P.Herschbach@tum.de 